\title{
A game-changer for hereditary cancer patients
}

\author{
Cite as: CMAJ 2017 June 19;189:E843-4. doi: 10.1503/cmaj.1095411
}

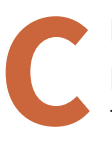

hiquita Hessels' uncle died of prostate cancer. Her sister had thyroid cancer and died at age 32 . Her mother, grandmother and greatgrandmother succumbed to breast cancer. An aunt survived bone and colon cancer, only to die of liver cancer. Most families would consider their family tree diseased, but for Nanaimo, British Columbia resident and breast cancer survivor Hessels it has another name: Li-Fraumeni syndrome (LFS).

Patients with LFS, a rare hereditary condition also known as the "ticking-time bomb disease," have a mutation to the TP53 tumour suppressor gene which leads to a more than $90 \%$ chance of developing cancer in their lifetime - in any part of the body and at any age. Each case is different, but some patients can experience six or seven different cancers before the age of 18 .

About one in 1000 to 5000 people live with LFS, according to Dr. David Malkin, a pediatric oncologist and internationally renowned LFS researcher at Toronto's SickKids Hospital, a number some researchers believe is underestimated because of underdiagnosis. He has developed a screening protocol - the "Toronto Protocol" - that is used across Canada and around the world to detect cancers as early as possible in people living with LFS, as well as those with other hereditary cancer predisposition syndromes.

Malkin published a paper in The Lancet Oncology detailing a longer follow-up on patients following his Toronto Protocol.

"The goal of the Toronto Protocol [was] to develop a surveillance protocol to detect tumours early, with the potential that one may be able to intervene sooner and reduce morbidity and mortality," said Malkin, noting that aspects of the protocol are now used to guide sur- veillance for early tumor detection in a variety of other hereditary cancer syndromes. "The challenges faced by these families is devastating."

The premise of the Toronto Protocol is simple: periodically screen patients using a series of biochemical assays and imaging studies (including mammography, colonoscopy, abdominal and pelvic ultrasound, and brain and whole body magnetic resonance imaging [MRI]). Malkin's paper demonstrated a five-year overall survival rate of $89 \%$ for the surveillance group as compared to less than $60 \%$ for the non-surveillance group.

Potential downsides of the surveillance protocol include cost (Malkin estimates $\$ 400$ to $\$ 5000$ per patient per year), exposure to radiation and/or unnecessary interventions, and the possibility for false

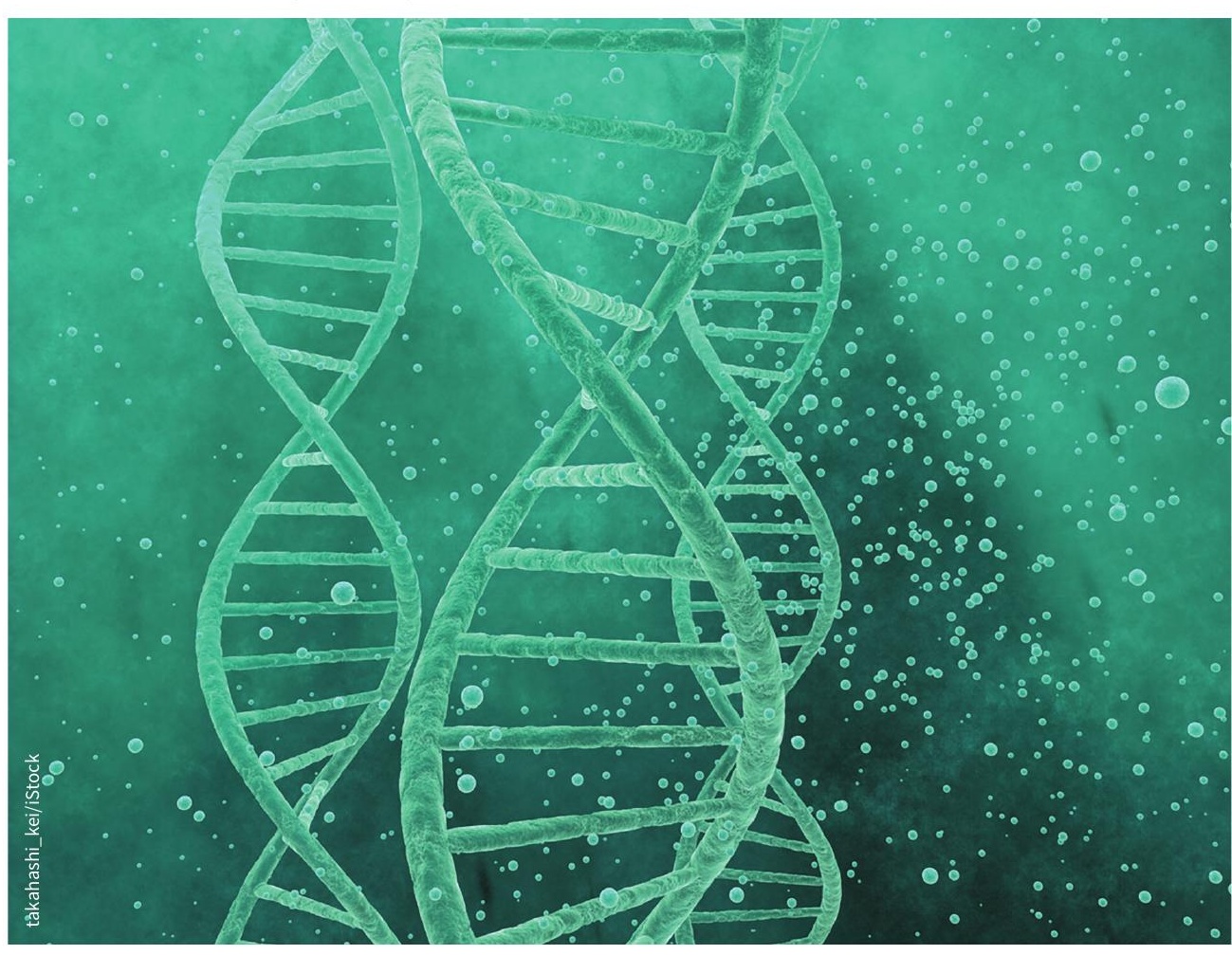

The Toronto protocol of intensive screening is designed to help patients with Li-Fraumeni syndrome. positives or overdiagnosis of benign tumours. However, given that LFS has a penetrance of $90 \%$, experts agree that the benefits of this screening protocol are compelling.

In fact, the excitement generated from the Toronto Protocol helped to spearhead a workshop sponsored by the American Association for Cancer Research in Boston in October 2016. Over 60 specialists from centres around the world met to develop a series of surveillance recommendations for over 50 distinct cancer predisposition syndromes. These included hereditary breast and ovarian cancer (BRCA1/ BRCA2), hereditary colon cancer syndromes (Lynch syndrome and familial adenomatous polyposis), von HippelLindau disease, Beckwith-Wiedemann 
syndrome, and DICER1 syndrome. The recommendations from the workshop will be published later in 2017 and researchers hope it will inform practitioners worldwide.

Malkin says that in light of the growing research on hereditary cancer syndromes, it's critical for family physicians to both identify at-risk patients and help them access the surveillance that could save their lives. This includes taking a good family history and updating it every few months, he added, noting that accurate information is the key to detecting patterns that may point toward familial cancer syndromes.
When it comes to living with hereditary cancer syndromes such as LFS, families like the Hessels say there is comfort in knowing they are being closely monitored. Chiquita's two sons, and her 29-year-old niece, were both recently found to have LFS; all four undergo regular screening under the Toronto Protocol.

"Information is a strong and powerful tool, and my family will benefit from regular screening," said Hessels. "If something shows up, at least it should be early stage and easier to manage and deal with."

The Toronto Protocol is currently publicly funded across Canada as an intensive screening program for patients with LFS.
However, some provinces such as B.C., do not fully cover the costs, meaning patients incur some expenses and potential lengthy wait times during the screening process. The $\$ 1600$ cost of full body MRIs, for example, are paid out of pocket by the Hessels family.

"I think we in BC are lagging behind in this area, and I hope and trust that those who have the power to make change do so, and upgrade to this model," said Hessels. "It's proven to save lives... you can be proactive and involved in your own care if you have some answers."

Brit Cooper-Jones and Katelyn

Verstraten, Vancouver, British Columbia 